DOI: $10.15393 /$ j3.art.2022.10431

UDC 517.988

I. K. Argyros, S. George, C. Argyros

\title{
A BALL COMPARISON BETWEEN EXTENDED MODIFIED JARRATT METHODS UNDER THE SAME SET OF CONDITIONS FOR SOLVING EQUATIONS AND SYSTEMS OF EQUATIONS
}

\begin{abstract}
In this paper, we compare the radii of convergence of Jarratt-type methods under the same set of conditions for solving nonlinear equations and systems of equations. Our convergence analysis is based on the first Fréchet derivative that only appears on the method. Numerical examples where the theoretical results are tested complete the paper.
\end{abstract}

Key words: Jarratt type method, Banach space, radious of convergence

2020 Mathematical Subject Classification: 65J20, 49M15, 74G20, 41A25

1. Introduction. In this paper we compare the convergence radii of Jarratt-type methods under the same set of conditions for solving nonlinear equation

$$
F(x)=0,
$$

where $F: \Omega \subset \mathcal{B}_{1} \longrightarrow \mathcal{B}_{2}$ is continuously Fréchet differentiable, $\mathcal{B}_{1}, \mathcal{B}_{2}$ are Banach spaces, and $\Omega$ is a nonempty convex set.

The methods under consideration in this paper are [14]:

$$
\begin{aligned}
y_{n} & =x_{n}-\frac{1}{2} F^{\prime}\left(x_{n}\right)^{-1} F\left(x_{n}\right), \\
z_{n} & \left.=x_{n}-\frac{2}{3} F^{\prime}\left(x_{n}\right)^{-1} F\left(x_{n}\right)\right), \\
x_{n+1} & =y_{n}+\left(F^{\prime}\left(x_{n}\right)-3 F^{\prime}\left(z_{n}\right)\right)^{-1}\left(F\left(x_{n}\right)+2 F\left(z_{n}\right)\right),
\end{aligned}
$$

and [13]

$$
y_{n}=x_{n}-\frac{2}{3} F^{\prime}\left(x_{n}\right)^{-1} F\left(x_{n}\right),
$$

(C) Petrozavodsk State University, 2022 


$$
\begin{aligned}
z_{n}= & x_{n}-\frac{1}{2}\left(3 F^{\prime}\left(y_{n}\right)-F^{\prime}\left(x_{n}\right)\right)^{-1}\left(3 F^{\prime}\left(y_{n}\right)+\right. \\
& \left.+F^{\prime}\left(x_{n}\right)\right) F^{\prime}\left(x_{n}\right)^{-1} F\left(x_{n}\right), \\
x_{n+1}= & z_{n}-2\left(3 F^{\prime}\left(y_{n}\right)-F^{\prime}\left(x_{n}\right)\right)^{-1} F\left(z_{n}\right) .
\end{aligned}
$$

The sixth-order convergence of iterative methods was, in general, obtained in [13], [14], respectively, when $\mathcal{B}_{1}=\mathcal{B}_{2}=\mathbb{R}^{m}$. Taylor expansions were used, and conditions up to the seven order derivative restrict applicability of the methods.

For example: Let $\mathcal{B}_{1}=\mathcal{B}_{2}=\mathbb{R}, \Omega=\left[-\frac{1}{2}, \frac{3}{2}\right]$. Define $f$ on $\Omega$ by

$$
f(t)=\left\{\begin{array}{cc}
t^{3} \log t^{2}+t^{5}-t^{4} & \text { if } t \neq 0 \\
0 & \text { if } t=0
\end{array}\right.
$$

Then, we have $t_{*}=1$,

$$
f^{\prime \prime \prime}(t)=6 \log t^{2}+60 t^{2}-24 t+22 .
$$

Obviously $f^{\prime \prime \prime}(t)$ is not bounded on $\Omega$. So, convergence of these methods is not guaranteed by the analysis in these papers. Our convergence analysis is based on the first Fréchet derivative that only appears on the method.

We also provide a computable radius of convergence, not given in [1-22]. This way, we locate a set of initial points for the convergence of the method. The numerical examples are chosen to show how the radii theoretically predicted are computed. In particular, the example given by (3) shows that earlier results cannot be used to show convergence of the method. Our results significantly extend the applicability of these methods and provide a new way of looking at iterative methods.

The article contains local convergence analysis in Section 2 and the numerical examples in Section 3.

2. Ball convergence. We develop real functions to be used in the ball-convergence analysis of method (1) and (2), respectively. Set $S=[0, \infty)$. Assume

(i) Equation

$$
\varphi_{0}(t)-1=0
$$

has the least solution $\rho_{0} \in S-\{0\}$ for some function $\varphi_{0}: S \longrightarrow S$ continuous and nondecreasing. Let $S_{0}=\left[0, \rho_{0}\right)$; 
(ii) equation

$$
\psi_{1}(t)-1=0
$$

has the least solution $r_{1} \in S_{0}-\{0\}$, for some functions $\varphi: S_{0} \longrightarrow S$ and $\varphi_{1}: S_{0} \longrightarrow S$ continuous and nondecreasing, where

$$
\psi_{1}(t)=\frac{\int_{0}^{1} \varphi((1-\theta) t) d \theta+\frac{1}{2} \int_{0}^{1} \varphi_{1}(\theta t) d \theta}{1-\varphi_{0}(t)} ;
$$

(iii) equation

$$
\psi_{2}(t)-1=0
$$

has the least solution $r_{2} \in S_{0}-\{0\}$, where

$$
\psi_{2}(t)=\frac{\int_{0}^{1} \varphi((1-\theta) t) d \theta+\frac{1}{3} \int_{0}^{1} \varphi_{1}(\theta t) d \theta}{1-\varphi_{0}(t)} ;
$$

(iv) equation

$$
p(t)-1=0,
$$

has the least positive solution $\rho_{p}$, where $p(t)=\frac{1}{3}\left(3 \varphi_{0}\left(\psi_{2}(t) t\right)+\varphi_{0}(t)\right)$. Let $\rho=\min \left\{\rho_{0}, \rho_{p}\right\}$ and $S_{1}=[0, \rho)$. Assume equation

$$
\psi_{3}(t)-1=0
$$

has the least solution $r_{3} \in(0, \rho)$, where

$$
\begin{array}{r}
\psi_{3}(t)=\frac{\int_{0}^{1} \varphi((1-\theta) t) d \theta}{1-\varphi_{0}(t)}+\frac{3\left(\varphi_{0}(t)+\varphi_{0}\left(\psi_{2}(t) t\right)\right) \int_{0}^{1} \varphi_{1}(\theta t) d \theta}{4\left(1-\varphi_{0}(t)\right)(1-p(t))}+ \\
+\frac{\int_{0}^{1} \varphi_{1}\left(\theta \psi_{2}(t) t\right) d \theta \psi_{2}(t)}{1-p(t)}
\end{array}
$$

The parameter $r$ defined by

$$
r=\min \left\{r_{k}\right\}, k=1,2,3,
$$

shall be shown to be a convergence radius for method (1). We have, by (4), that for all $t \in[0, r)$

$$
0 \leqslant \varphi_{0}(t)<1,
$$




$$
0 \leqslant p(t)<1
$$

and

$$
0 \leqslant \psi_{k}(t)<1 .
$$

The notation $C(x, a), \bar{C}(x, a)$ is used for the open and closed balls, respectively, in $\mathcal{B}_{1}$ with center $x \in \mathcal{B}_{1}$ and of radius $a>0$.

The conditions (A) are needed with functions $\varphi_{0}, \varphi$, and $\varphi_{1}$ as defined previously. Further, assume:

(a1) $F: \Omega \subset \mathcal{B}_{1} \longrightarrow \mathcal{B}_{2}$ is Fréchet-continuously differentiable; there exists a simple $x_{*} \in \Omega$ such that $F\left(x_{*}\right)=0$.

(a2) For each $x \in \Omega$

$$
\left\|F^{\prime}\left(x_{*}\right)^{-1}\left(F^{\prime}(x)-F^{\prime}\left(x_{*}\right)\right)\right\| \leqslant \varphi_{0}\left(\left\|x-x_{*}\right\|\right) .
$$

Set $\Omega_{0}=\Omega \cap C\left(x_{*}, \rho_{0}\right)$.

(a3) For each $x, y \in \Omega_{0}$,

$$
\begin{gathered}
\left\|F^{\prime}\left(x_{*}\right)^{-1}\left(F^{\prime}(y)-F^{\prime}(x)\right)\right\| \leqslant \varphi(\|y-x\|), \\
\left\|F^{\prime}\left(x_{*}\right)^{-1} F^{\prime}(x)\right\| \leqslant \varphi_{1}\left(\left\|x-x_{*}\right\|\right) .
\end{gathered}
$$

(a4) $\bar{C}\left(x_{*}, \gamma\right) \subset \Omega$ for some $\gamma>0$ to be determined.

(a5) There exists $b \geqslant r$ such that $\int_{0}^{1} \varphi_{0}(\theta b) d \theta<1$. Set $\Omega_{1}=\Omega \cap \bar{T}\left(x_{*}, b\right)$.

The ball convergence of method (1) follows, based on conditions (A).

Theorem 1. Assume conditions (A) with $\gamma=r$ hold and choose $x_{0} \in C\left(x_{*}, r\right)-\left\{x_{*}\right\}$. Then, iteration $\left\{x_{n}\right\}$ generated by method (1) is well-defined in $C\left(x_{*}, r\right)$, remains in $C\left(x_{*}, r\right)$ for all $n=0,1,2, \ldots$, with $\lim _{n \rightarrow \infty} x_{n}=x_{*}$,

$$
\begin{gathered}
\left\|y_{n}-x_{*}\right\| \leqslant \psi_{1}\left(\left\|x_{n}-x_{*}\right\|\right)\left\|x_{n}-x_{*}\right\| \leqslant\left\|x_{n}-x_{*}\right\|<r \\
\left\|z_{n}-x_{*}\right\| \leqslant \psi_{2}\left(\left\|x_{n}-x_{*}\right\|\right)\left\|x_{n}-x_{*}\right\| \leqslant\left\|x_{n}-x_{*}\right\|,
\end{gathered}
$$

and

$$
\left\|x_{n+1}-x_{*}\right\| \leqslant \psi_{3}\left(\left\|x_{n}-x_{*}\right\|\left\|x_{n}-x_{*}\right\| \leqslant\left\|x_{n}-x_{*}\right\|,\right.
$$

where the functions $\psi_{k}, k=1,2,3$ are given previously and $r$ is given by (6). Moreover, $x_{*}$ the only solution of equation $F(x)=0$ in the set $\Omega_{1}$ given in (a5). 
Proof. Estimates (8) - (10) are proved by induction on $i$. Using (4), (5), (a1) and (a2), we get, in turn, for $u \in C\left(x_{*}, r\right)-\left\{x_{*}\right\}$ :

$$
\left\|F^{\prime}\left(x_{*}\right)^{-1}\left(F^{\prime}(u)-F^{\prime}\left(x_{*}\right)\right)\right\| \leqslant \varphi_{0}\left(\left\|u-x_{*}\right\|\right) \leqslant \varphi_{0}(r)<1 .
$$

Then, the Banach lemma on invertible operators [8] and (11) gives $F^{\prime}(u)^{-1} \in L\left(\mathcal{B}_{2}, \mathcal{B}_{1}\right)$, with

$$
\left\|F^{\prime}(u)^{-1} F^{\prime}\left(x_{*}\right)\right\| \leqslant \frac{1}{1-\varphi_{0}\left(\left\|u-x_{*}\right\|\right)} .
$$

It follows that iterates $y_{0}$ and $z_{0}$ are well-defined by method (1) for $n=0$. We can also write

$$
y_{0}-x_{*}=\left(x_{0}-x_{*}-F^{\prime}\left(x_{0}\right)^{-1} F\left(x_{0}\right)\right)+\frac{1}{2} F^{\prime}\left(x_{0}\right)^{-1} F^{\prime}\left(x_{0}\right)
$$

and

$$
z_{0}-x^{*}=x_{0}-x^{*}-\frac{2}{3} F^{\prime}\left(x_{0}\right)^{-1} F\left(x_{0}\right) .
$$

In view of (4), (7) (for $k=1,2),(\mathrm{a} 3),(12)$ (for $u=x_{0}$ ) and (13), we have, in turn:

$$
\begin{aligned}
& \left\|y_{0}-x_{*}\right\| \leqslant\left\|F^{\prime}\left(x_{0}\right)^{-1} F\left(x_{*}\right)\right\| \times \\
& \quad \times \| \int_{0}^{1} F^{\prime}\left(x_{*}\right)^{-1}\left(F^{\prime}\left(x_{*}+\theta\left(x_{0}-x_{*}\right)\right)-F^{\prime}\left(x_{0}\right)\right) d \theta\left(x_{0}-x_{*}\right)+ \\
& \quad+\frac{1}{2}\left\|F^{\prime}\left(x_{0}\right)^{-1} F\left(x_{*}\right)\right\|\left\|F^{\prime}\left(x_{0}\right)^{-1} F\left(x_{0}\right)\right\| \leqslant \\
& \leqslant \frac{\left(\int_{0}^{1} \varphi\left((1-\theta)\left\|x_{0}-x_{*}\right\|\right) d \theta+\frac{1}{2} \int_{0}^{1} \varphi_{1}\left(\theta\left\|x_{0}-x_{*}\right\|\right) d \theta\right.}{1-\varphi_{0}\left(\left\|x_{0}-x_{*}\right\|\right)}\left\|x_{0}-x_{*}\right\|= \\
& =\psi_{1}\left(\left\|x_{0}-x_{*}\right\|\right)\left\|x_{0}-x_{*}\right\| \leqslant\left\|x_{0}-x_{*}\right\|<r,
\end{aligned}
$$

and

$$
\begin{aligned}
& \left\|z_{0}-x_{*}\right\| \leqslant \\
& \quad \leqslant\left[\frac{\left.\int_{0}^{1} \varphi\left((1-\theta)\left\|x_{0}-x_{*}\right\|\right) d \theta+\frac{1}{3} \int_{0}^{1} \varphi_{1}\left(\theta\left\|x_{0}-x_{*}\right\|\right) d \theta\right)}{1-\varphi_{0}\left(\left\|x_{0}-x_{*}\right\|\right)}\right]\left\|x_{0}-x_{*}\right\| \leqslant
\end{aligned}
$$




$$
\leqslant \psi_{2}\left(\left\|x_{0}-x_{*}\right\|\right)\left\|x_{0}-x_{*}\right\| \leqslant\left\|x_{0}-x_{*}\right\| ;
$$

this shows that $y_{0}, z_{0} \in C\left(x_{*}, r\right)$ and (8), (9) hold for $n=0$, respectively.

Next, we show that $\left(F^{\prime}\left(x_{0}\right)-3 F^{\prime}\left(z_{0}\right)\right)$ is invertible; this will imply the existence of iterate $x_{1}$. Indeed, using (4), (6) and (16), we obtain, in turn, that

$$
\begin{aligned}
& \left\|\left(-2 F^{\prime}\left(x_{*}\right)\right)^{-1}\left(F^{\prime}\left(x_{0}\right)-3 F^{\prime}\left(z_{0}\right)+2 F^{\prime}\left(x_{*}\right)\right)\right\| \leqslant \\
\leqslant & \frac{1}{2}\left(3\left\|F^{\prime}\left(x_{*}\right)^{-1}\left(F^{\prime}\left(z_{0}\right)-F^{\prime}\left(x_{*}\right)\right)\right\|+\left\|F^{\prime}\left(x_{*}\right)^{-1}\left(F^{\prime}\left(x_{0}\right)-F\left(x_{*}\right)\right)\right\|\right) \leqslant \\
\leqslant & \frac{1}{3}\left(3 \varphi_{0}\left(\left\|z_{0}-x_{*}\right\|\right)+\varphi_{0}\left(\left\|x_{0}-x_{*}\right\|\right)\right) \leqslant \\
\leqslant & p\left(\left\|x_{0}-x_{*}\right\| \leqslant p(r)<1,\right.
\end{aligned}
$$

SO

$$
\left\|\left(F^{\prime}\left(x_{0}\right)-3 F^{\prime}\left(z_{0}\right)\right)^{-1} F^{\prime}\left(x_{*}\right)\right\| \leqslant \frac{1}{2\left(1-p\left(\left\|x_{0}-x_{*}\right\|\right)\right)} .
$$

It follows from (4), (7) (for $k=3$ ), (15), (16) and (18), and

$$
\begin{aligned}
x_{1}-x_{*}= & x_{0}-x_{*}-F^{\prime}\left(x_{0}\right)^{-1} F\left(x_{0}\right)+ \\
& +\frac{1}{2} F^{\prime}\left(x_{0}\right)^{-1} F\left(x_{0}\right)+ \\
& +\left(F^{\prime}\left(x_{0}\right)-3 F\left(z_{0}\right)\right)^{-1} F^{\prime}\left(x_{0}\right)+2\left(F^{\prime}\left(x_{0}\right)-3 F^{\prime}\left(z_{0}\right)\right)^{-1} F\left(z_{0}\right)= \\
= & x_{0}-x_{*}-F^{\prime}\left(x_{0}\right)^{-1} F\left(x_{0}\right)+ \\
& +\frac{3}{2} F^{\prime}\left(x_{0}\right)^{-1}\left(F^{\prime}\left(z_{0}\right)-F^{\prime}\left(x_{0}\right)\right)\left(F^{\prime}\left(x_{0}\right)-3 F^{\prime}\left(z_{0}\right)\right)^{-1} F\left(x_{0}\right)+ \\
& +2\left(F^{\prime}\left(x_{0}\right)-3 F^{\prime}\left(z_{0}\right)\right)^{-1} F\left(z_{0}\right) .
\end{aligned}
$$

We have, in turn,

$$
\begin{gathered}
\left\|x_{1}-x_{*}\right\| \leqslant \\
\leqslant \frac{\int_{0}^{1} \varphi\left((1-\theta)\left\|x_{0}-x_{*}\right\|\right) d \theta\left\|x_{0}-x_{*}\right\|}{1-\varphi_{0}\left(\left\|x_{0}-x_{*}\right\|\right)}+ \\
+\frac{3\left(\varphi_{0}\left(\left\|x_{0}-x_{*}\right\|\right)+\varphi_{0}\left(\left\|z_{0}-x_{*}\right\|\right)\right) \int_{0}^{1} \varphi_{1}\left(\theta\left\|x_{0}-x_{*}\right\|\right) d \theta\left\|x_{0}-x_{*}\right\|}{4\left(1-\varphi_{0}\left(\left\|x_{0}-x_{*}\right\|\right)\right)\left(1-p\left(\left\|x_{0}-x_{*}\right\|\right)\right)}+ \\
+\frac{\left(\varphi_{1}\left(\theta\left\|z_{0}-x_{*}\right\|\right) d \theta\left\|z_{0}-x_{*}\right\|\right.}{1-p\left(\left\|x_{0}-x_{*}\right\|\right)} \leqslant
\end{gathered}
$$




$$
\leqslant \psi_{3}\left(\left\|x_{0}-x_{*}\right\|\right)\left\|x_{0}-x_{*}\right\| \leqslant\left\|x_{0}-x_{*}\right\|,
$$

showing (10) for $n=0$ and $x_{1} \in C\left(x_{*}, r_{*}\right)$. Then, repalce $x_{0}, y_{0}, z_{0}, x_{1}$ by $x_{i}, y_{i}, z_{i}, x_{i+1}$ in the preceding calculations to complete the induction for items (8)-(10). In view of the estimation

$$
\left\|x_{i+1}-x_{*}\right\| \leqslant \beta\left\|x_{i}-x_{*}\right\|<r
$$

where $\beta=\psi_{3}\left(\left\|x_{0}-x_{*}\right\|\right) \in[0,1)$, we conclude that $\lim _{i \rightarrow \infty} x_{i}=x_{*}$ and $x_{i+1} \in C\left(x_{*}, r_{*}\right)$. The uniqueness part is left to complete the proof. Consider $v \in \Omega_{1}$ with $F(v)=0$. Let $G=\int_{0}^{1} F^{\prime}\left(x_{*}+\theta\left(v-x_{*}\right)\right) d \theta$. Then, using (a2) and (a5), we obtain

$$
\left\|F^{\prime}\left(x_{*}\right)^{-1}\left(G-F^{\prime}\left(x_{*}\right)\right)\right\| \leqslant \int_{0}^{1} \varphi_{0}\left(\theta \| x_{*}-v\right) \| d \theta \leqslant \int_{0}^{1} \varphi_{0}(\theta b) d \theta<1,
$$

so $G^{-1} \in L\left(\mathcal{B}_{2}, \mathcal{B}_{1}\right)$. Consequently, from $0=F(v)-F\left(x_{*}\right)=G\left(v-x_{*}\right)$, we obtain $v=x_{*}$.

\section{Remark.}

1. In view of (a2) and the estimate

$$
\begin{aligned}
\| F^{\prime}\left(x^{*}\right)^{-1} & F^{\prime}(x)\|=\| F^{\prime}\left(x^{*}\right)^{-1}\left(F^{\prime}(x)-F^{\prime}\left(x^{*}\right)\right)+I \| \leqslant \\
& \leqslant 1+\left\|F^{\prime}\left(x^{*}\right)^{-1}\left(F^{\prime}(x)-F^{\prime}\left(x^{*}\right)\right)\right\| \leqslant 1+\varphi_{0}\left(\left\|x-x^{*}\right\|\right)
\end{aligned}
$$

the second condition in (h3) can be dropped and $\varphi_{1}$ can be replaced by

$$
\varphi_{1}(t)=1+\varphi_{0}(t)
$$

or

$$
\varphi_{1}(t)=1+\varphi_{0}\left(\rho_{0}\right)
$$

since $t \in\left[0, \rho_{0}\right)$.

2. The results obtained here can be used for operators $F$ satisfying autonomous differential equations [2] of the form

$$
F^{\prime}(x)=P(F(x))
$$

where $P$ is a continuous operator. Since $F^{\prime}\left(x^{*}\right)=P\left(F\left(x^{*}\right)\right)=P(0)$, we can apply the results without actually knowing $x^{*}$. For example, let $F(x)=e^{x}-1$. Then we can choose $P(x)=x+1$. 
3. Let $\varphi_{0}(t)=L_{0} t$, and $\varphi(t)=L t$. In [2], [3], we showed that $r_{A}=\frac{2}{2 L_{0}+L}$ is the convergence radius of Newton's method:

$$
x_{n+1}=x_{n}-F^{\prime}\left(x_{n}\right)^{-1} F\left(x_{n}\right) \text { for each } n=0,1,2, \ldots
$$

under the conditions (a1)-(a3). It follows from the definition that the convergence radius $r$ of the method (1) cannot be larger than the convergence radius $r_{A}$ of the second-order Newton's method (22). As already noted in [2], [3], $r_{A}$ is at least as large as the convergence radius given by Rheinboldt [18]

$$
r_{R}=\frac{2}{3 L_{1}}
$$

where $L_{1}$ is the Lipschitz constant on $D$. The same value for $r_{R}$ was given by Traub [19]. In particular, for $L_{0}<L_{1}$ we have

$$
r_{R}<r_{A}
$$

and

$$
\frac{r_{R}}{r_{A}} \rightarrow \frac{1}{3} \text { as } \frac{L_{0}}{L_{1}} \rightarrow 0 .
$$

That is, the radius of convergence $r_{A}$ is at most three times larger than Rheinboldt's.

4. We can compute the computational order of convergence (COC) defined by

$$
\xi=\ln \left(\frac{\left\|x_{n+1}-x^{*}\right\|}{\left\|x_{n}-x^{*}\right\|}\right) / \ln \left(\frac{\left\|x_{n}-x^{*}\right\|}{\left\|x_{n-1}-x^{*}\right\|}\right)
$$

or the approximate computational order of convergence

$$
\xi_{1}=\ln \left(\frac{\left\|x_{n+1}-x_{n}\right\|}{\left\|x_{n}-x_{n-1}\right\|}\right) / \ln \left(\frac{\left\|x_{n}-x_{n-1}\right\|}{\left\|x_{n-1}-x_{n-2}\right\|}\right) .
$$

The ball convergence of method (2) follows in a similar way. Let $\bar{\psi}_{1}=\psi_{2}$ and define functions

$$
\bar{\psi}_{2}(t)=\frac{\int_{0}^{1} \varphi((1-\theta) t) d \theta}{1-\varphi_{0}(t)}+\frac{3}{4} \frac{\left(\varphi_{0}(t)+\varphi_{0}\left(\bar{\psi}_{1}(t) t\right)\right)}{\left(1-\varphi_{0}(t)\right)(1-q(t))},
$$




$$
\left.\left.q(t)=\frac{1}{2}\left(\varphi_{0}(t)+3 \varphi_{0}\left(\bar{\psi}_{1}\right) t\right) t\right)\right)
$$

and

$$
\begin{aligned}
\bar{\psi}_{3}(t)= & {\left[\frac{\int_{0}^{1} \varphi\left((1-\theta) \bar{\psi}_{2}(t) t\right) d \theta}{1-\varphi_{0}\left(\bar{\psi}_{2}(t) t\right)}+\right.} \\
& \left.+\frac{\left(3 \varphi_{0}\left(\bar{\psi}_{1}(t) t\right)+2 \varphi_{0}\left(\bar{\psi}_{2}(t) t\right)+\varphi_{0}(t)\right) \int_{0}^{1} \varphi_{1}\left(\theta \bar{\psi}_{2}(t) t\right) d \theta}{2\left(1-\varphi_{0}\left(\bar{\psi}_{2}(t) t\right)\right)(1-q(t))}\right] \bar{\psi}_{2}(t) .
\end{aligned}
$$

Assume the equations

$$
\bar{\psi}_{2}(t)-1=0 \text { and } \bar{\psi}_{3}(t)-1=0
$$

have the minimal positive solutions $\bar{r}_{2}$ and $\bar{r}_{3}$ in $A_{0}$, respectively. Let

$$
\bar{r}=\min \left\{r_{2}, \bar{r}_{2}, \bar{r}_{3}\right\} .
$$

We need the estimates under conditions (A) for $\gamma=\bar{r}$ :

$$
\begin{gathered}
\left\|y_{n}-x_{*}\right\| \leqslant \psi_{2}\left(\left\|x_{n}-x_{*}\right\|\right)\left\|x_{n}-x_{*}\right\|= \\
=\bar{\psi}_{1}\left(\left\|x_{n}-x_{*}\right\|\right)\left\|x_{n}-x_{*}\right\| \leqslant\left\|x_{n}-x_{*}\right\|<\bar{r}, \\
z_{n}-x_{*}=x_{n}-x_{*}-F^{\prime}\left(x_{n}\right)^{-1} F\left(x_{n}\right)+ \\
+\left[I-\frac{1}{2}\left(3\left(F^{\prime}\left(y_{n}\right)-F^{\prime}\left(x_{n}\right)\right)^{-1}\left(3 F^{\prime}\left(y_{n}\right)+F^{\prime}\left(x_{n}\right)\right)\right] F^{\prime}\left(x_{0}\right)^{-1} F\left(x_{n}\right)=\right. \\
=x_{n}-x_{*}-F^{\prime}\left(x_{n}\right)^{-1} F\left(x_{n}\right)+ \\
+\frac{3}{2}\left(3 F^{\prime}\left(y_{n}\right)-F^{\prime}\left(x_{n}\right)\right)^{-1}\left(F^{\prime}\left(y_{n}\right)-F^{\prime}\left(x_{n}\right)\right) F^{\prime}\left(x_{n}\right)^{-1} F\left(x_{n}\right),
\end{gathered}
$$

SO

$$
\begin{aligned}
& \left\|z_{n}-x_{*}\right\| \leqslant\left[\frac{\int_{0}^{1} \varphi\left((1-\theta)\left\|x_{n}-x_{*}\right\|\right) d \theta}{1-\varphi_{0}\left(\left\|x_{n}-x_{*}\right\|\right)}+\right. \\
& \left.+\frac{3}{4} \frac{\left(\varphi_{1}\left(\left\|y_{n}-x_{*}\right\|\right)+\varphi_{0}\left(\left\|x_{n}-x_{*}\right\|\right)\right) \int_{0}^{1} \varphi_{1}\left(\theta\left\|x_{n}-x_{*}\right\|\right) d \theta}{\left(1-\varphi_{0}\left(\left\|x_{n}-x_{*}\right\|\right)\right)\left(1-q\left(\left\|x_{n}-x_{*}\right\|\right)\right)}\right]\left\|x_{n}-x_{*}\right\| \leqslant
\end{aligned}
$$




$$
\begin{aligned}
\leqslant \bar{\psi}_{2}\left(\left\|x_{n}-x_{*}\right\|\right)\left\|x_{n}-x_{*}\right\| \leqslant\left\|x_{n}-x_{*}\right\|<\bar{r}, \\
\begin{aligned}
& x_{n+1}-x_{*}=z_{n}-x_{*}-F^{\prime}\left(z_{n}\right)^{-1} F\left(z_{n}\right)+ \\
&+\left(F^{\prime}\left(z_{n}\right)^{-1}-2\left(3 F^{\prime}\left(y_{n}\right)-F^{\prime}\left(x_{n}\right)\right)\right)^{-1} F\left(z_{n}\right)= \\
&=z_{n}-x_{*}-F^{\prime}\left(z_{n}\right)^{-1} F\left(z_{n}\right)+F^{\prime}\left(z_{n}\right)^{-1}(\left.3 F^{\prime}\left(y_{n}\right)-F^{\prime}\left(x_{n}\right)-2 F^{\prime}\left(z_{n}\right)\right) \times \\
& \times\left(3 F^{\prime}\left(y_{n}\right)-F^{\prime}\left(x_{n}\right)\right)^{-1} F\left(z_{n}\right),
\end{aligned}
\end{aligned}
$$

SO

$$
\begin{aligned}
\left\|x_{n+1}-x_{*}\right\| \leqslant & {\left[\frac{\int_{0}^{1} \varphi\left((1-\theta)\left\|z_{n}-x_{*}\right\|\right) d \theta}{1-\varphi_{0}\left(\left\|z_{n}-x_{*}\right\|\right)}+\right.} \\
& +\frac{\left(3 \varphi_{0}\left(\left\|y_{n}-x_{*}\right\|\right)+2 \varphi_{0}\left(\left\|z_{n}-x_{*}\right\|\right)+\varphi_{0}\left(\left\|x_{n}-x_{*}\right\|\right)\right)}{\left(1-\varphi_{0}\left(\left\|z_{n}-x_{*}\right\|\right)\right)\left(1-q\left(\left\|x_{n}-x_{*}\right\|\right)\right)} \times \\
& \left.\times \int_{0}^{1} \varphi_{1}\left(\theta\left\|z_{n}-x_{*}\right\|\right) d \theta\right]\left\|z_{n}-x_{*}\right\| \leqslant \\
\leqslant & \bar{\psi}_{3}\left(\left\|x_{n}-x_{*}\right\|\right)\left\|x_{n}-x_{*}\right\| \leqslant\left\|x_{n}-x_{*}\right\|,
\end{aligned}
$$

where we also used as in (18) (for $y_{n}=z_{n}$ )

$$
\left\|\left(3 F^{\prime}\left(y_{n}\right)-F^{\prime}\left(x_{n}\right)\right)^{-1} F^{\prime}\left(x_{*}\right)\right\| \leqslant \frac{1}{2\left(1-q\left(\left\|x_{n}-x_{*}\right\|\right)\right)} .
$$

Hence, we arrive at:

Theorem 2. Assume conditions (A) with $\gamma=\bar{r}$ hold and choose $x_{0} \in C\left(x_{*}, \bar{r}_{*}\right)-\left\{x_{*}\right\}$. Then, the conclusions of Theorem 1 hold for method (2) with $\bar{\psi}_{2}, \bar{\psi}_{3}, \bar{r}$ replacing $\psi_{2}, \psi_{3}$ and $r$, respectively.

3. Numerical Example. Consider the kinematic system

$$
F_{1}^{\prime}(x)=e^{x}, F_{2}^{\prime}(y)=(e-1) y+1, F_{3}^{\prime}(z)=1
$$

with $F_{1}(0)=F_{2}(0)=F_{3}(0)=0$. Let $F=\left(F_{1}, F_{2}, F_{3}\right)$. Let $\mathcal{B}_{1}=\mathcal{B}_{2}=\mathbb{R}^{3}$, $\Omega=\bar{U}(0,1), x_{*}=(0,0,0)^{T}$. Define the function $F$ on $\Omega$ for $w=(x, y, z)^{T}$ by

$$
F(w)=\left(e^{x}-1, \frac{e-1}{2} y^{2}+y, z\right)^{T} .
$$


Then we get

$$
F^{\prime}(v)=\left[\begin{array}{ccc}
e^{x} & 0 & 0 \\
0 & (e-1) y+1 & 0 \\
0 & 0 & 1
\end{array}\right]
$$

so $\varphi_{0}(t)=(e-1) t, \varphi(t)=e^{\frac{1}{e-1}} t, \varphi_{1}(t)=e^{\frac{1}{e-1}}$. Then the radii are

$$
r_{1}=0.0402645, r_{2}=0.154407, r_{3}=0.688759, \bar{r}_{2}=0.138442, \bar{r}_{3}=0.112838 \text {; }
$$

so, we conclude that $r=r_{1}$ and $\bar{r}=\bar{r}_{3}$.

\section{References}

[1] Abbasbandy S., Bakhtiari P., Cordoro A., Torregrosa J. R., Lofti T. New efficient methods for solving nonlinear systems of equations with arbitrary even order. Appl. Math. Comput., 2016, vol. 287, 288, pp. 94-103.

DOI: https://doi.org/10.1016/j.amc.2016.04.038

[2] Argyros I. K. A unifying local-semilocal convergence analysis and applications for two-point Newton-like methods in Banach spaces. J. Math. Anal. Appl., 2004, vol. 298, pp. 374-397.

DOI: https://doi.org/10.1016/j.jmaa.2004.04.008

[3] Argyros I. K. Convergence and Applications of Newton-Type Iterations. Springer-Verlag, New York, 2008.

[4] Argyros I. K. A semilocal convergence analysis for directional Newton methods. Math. Comp., 2011, vol. 80, pp. 327-343.

https://doi.org/10.1090/S0025-5718-2010-02398-1

[5] Argyros I. K. Computational Theory of Iterative Methods, Series: Studies in Computational Mathematics. 15, Mditors: Chui C. K. and Wuytack L. Mlsevier Publ. Company, New York, 2007.

[6] Argyros I. K., Magreñán A. A. Iterative method and their dynamics with applications. CRC Press, New York, USA, 2017.

[7] Argyros I. K., George S., Magreñán A. A. Local convergence for multipoint- parametric Chebyshev-Halley-type method of higher convergence order. J. Comput. Appl. Math., 2015, vol. 282, pp. 215-224.

DOI: https://doi.org/10.1016/j.cam.2014.12.023

[8] Argyros I. K., Magreñán A. A. A study on the local convergence and the dynamics of Chebyshev-Halley-type methods free from second derivative. Numer. Algorithms, 2015, vol. 71, pp. 1-23.

DOI: https://doi.org/10.1007/s11075-015-9981-x 
[9] Argyros, I. K., George S. On the complexity of extending the convergence region for Traub's method. Journal of Complexity, 2020, vol. 56, 101423.

DOI: https://doi.org/10.1016/j.jco.2019.101423

[10] Argyros, I. K., George S. Mathematical modeling for the solution of equations and systems of equations with applications. Volume-IV, Nova Publishes, NY, 2020.

[11] Babajee D. K. R., Dauhoo M. Z., Darvishi M. T., Karami A., Barati A. Analysis of two Chebyshev-like third order methods free from second derivatives for solving systems of nonlinear equations. J. Comput. Appl. Math., 2010, vol. 233, pp. $2002-2012$.

http://dx.doi.org/10.1016/j.cam.2009.09.035

[12] Babajee D. K. R., Dauhoo M. Z., Darvishi M. T., Karami A., Barati A. A note on the local convergence of iterative methods based on Adomian decomposition method and 3-node quadrature rule. Applied Mathematics and Computation, 2008, vol. 200, no. 1, pp. 452-458.

DOI: https://doi.org/10.1016/j.amc.2007.11.009

[13] Cordero A., Hueso, J. J., Martinez E., Torregrosa J. R. A. A modified Newton-Jarratt's composition. Numer.Algor., 2010, vol. 55, pp. 87-99.

DOI: https://doi.org/10.1007/s11075-009-9359-z

[14] Cordero A., Torregrosa J. R. A., Vassileva M. P. Increasing the order of convergence of iterative methods for solving nonlinear systems. J. Comput. Appl. Math., 2012, vol. 252, pp. 86-94.

DOI: https://doi.org/10.1016/j.cam.2012.11.024

[15] Darvishi M. T. A two step high order Newton like method for solving systems of nonlinear equations. Int. J. Pure Appl. Math., 2009, vol. 57, pp. $543-555$.

[16] Darvishi M. T. Some three-step iterative methods free from second order derivative for finding solutions of systems of nonlinear equations. International Journal of Pure and Applied Mathematics, 2009, vol. 57, no. 4, pp. $557-573$.

[17] Grau-Sáchez M, Grau A., Noguera M. Ostrowski type methods for solving systems of nonlinear equations. Appl. Math. Comput., 2011, vol. 218, pp. 2377 -2385. DOI: http://doi.org/10.1016/j.amc.2011.08.011

[18] Jaiswal J. P. Semilocal convergnece of an eighth-order method in Banach spaces and its computational efficiency. Numer.Algorithms, 2016, vol. 71, pp. 933-951. DOI: https://doi.org/10.1007/s11075-015-0031-5

[19] Jaiswal J. P. Analysis of semilocal convergence in Banach spaces under relaxed condition and computational efficiency. Numer. Anal. Appl., 2017, vol. 10, pp. $129-139$. 
[20] Regmi S., Argyros I. K. Undergraduate Research at Cameron University on Iterative Procedures in Banach and Other Spaces, Nova Science Publisher, NY, 2019.

[21] Rheinboldt W. C. An adaptive continuation process for solving systems of nonlinear equations, In: Mathematical models and numerical methods (A. N. Tikhonov et al. eds.) Banach Center, Warsaw Poland, 1977, pub.3, pp. $129-142$.

[22] Traub J. F. Iterative Methods for the Solution of Mquations. Prentice-Hall, Mnglewood Cliffs, 1964.

Received June 10, 2021.

In revised form, August 11, 2021.

Accepted August 15, 2021.

Published online August 25, 2021.

Ioannis K. Argyros ${ }^{a}$

iargyros@cameron.edu

Santhosh George ${ }^{b}$

sgeorge@nitk.edu.in

Christopher $\operatorname{Argyros}^{a}$

christopher.argyros@cameron.edu

a. Cameron University 2800 W. Gore Blvd. Lawton, OK 73505-6377, USA

b. National Institute of Technology Karnataka, India-575 025 